Editorial

\title{
Mechanical Properties in Nuclear Installation and the Relevant Measurement Methods
}

\author{
Yan Yang, ${ }^{1}$ Alejandro Clausse, ${ }^{2}$ Leon Cizelj, ${ }^{3}$ Xing Chen, ${ }^{4}$ and Parashuram Sahoo ${ }^{5}$ \\ ${ }^{1}$ Chongqing University of Technology, Chongqing, China \\ ${ }^{2}$ National University of Central Buenos Aires, Buenos Aires, Argentina \\ ${ }^{3}$ Jožef Stefan Institute, Ljubljana, Slovenia \\ ${ }^{4}$ University of Utah, Salt Lake City, UT, USA \\ ${ }^{5}$ Indira Gandhi Centre for Atomic Research, Tamil Nadu, India
}

Correspondence should be addressed to Yan Yang; yangyan@cqut.edu.cn

Received 26 October 2016; Accepted 27 October 2016

Copyright (c) 2016 Yan Yang et al. This is an open access article distributed under the Creative Commons Attribution License, which permits unrestricted use, distribution, and reproduction in any medium, provided the original work is properly cited.

The application of nuclear technology is currently spreading over the world. Given the possibility of considerable risks, the lifetime performance and especially the safety of nuclear installation are raising large concerns to human society. Therefore, the assessment of mechanical properties and development of relevant measurement methods in nuclear installations are becoming extraordinarily important and are being intensively investigated. This field of research usually covers a wide range of subtopics, with the main focus on both fundamental sciences and advanced technologies for boosting the study of mechanical properties of nuclear installation and its related measurement. For example, fracture mechanics is useful to predict the temporal and long-term reliability of nuclear reactors under aggressive loads. Advanced functional materials (e.g., alloys and thermal barriers coatings) with superior thermal and mechanical resistance to harsh environment can be applied in nuclear installations. Also, it is of significant interest to analyze and characterize these materials by using state-of-the-art measurement methods.

Following up with the special issue "Advanced Measuring (Instrumentation) Methods for Nuclear Installations" published in the year of 2012, this special issue continues to demonstrate the most recent research progress in mechanical properties in nuclear installations and their relevant measurement methods. Six papers are included in this special issue covering a wide range of nuclear installation related topics.

In the paper "Effects of a Mixed Zone on TGO Displacement Instabilities of Thermal Barrier Coatings at High Temperature in Gas-Cooled Fast Reactors," thermally grown oxide has been studied as suitable protective coatings, offering thermal insulation, heat resistance, and corrosion resistance for Generation IV nuclear energy systems. The effects of mixed zone on the displacement instability of thermally grown oxide were assessed. Quantitative data show that mixed zone formation induces an enormous stress in thermally grown oxide, resulting in a sharp change of displacement compared to the alumina layer. The displacement instability increases with an increase in the mixed zone growth rate, growth strain, and thickness. Thus, the formation of a mixed zone accelerates the failure of thermally grown oxide, which is in agreement with previous experimental observations. These results provide data for the understanding of thermal barrier coatings failure mechanisms associated with mixed zone formation and of how to prolong working life of surface coating for gas cooled fast reactors.

The paper "Development of a Composite Technique for Preconditioning of 41Cr4 Steel Used as Gear Material: Examination of Its Microstructural Characteristics and Properties" reports a new composite technique to process commercial $41 \mathrm{Cr} 4$ steel. In this method, an intermediate layer was introduced firstly at the $41 \mathrm{Cr} 4$ steel surface by traditional carburizing and nitriding. Then a hard $\mathrm{Cr}$ coating was brush-plated on the intermediate layer. Finally, the coating layer was modified by high current pulsed electron beam (HCPEB), followed by quenching and subsequent tempering treatment. The microstructure, mechanical properties, and fracture behavior were characterized. The results show that a nanocrystalline $\mathrm{Cr}$ coating is formed at the $41 \mathrm{Cr} 4$ steel 
surface by the treatment of the new composite technique. Such nanocrystalline $\mathrm{Cr}$ coating has acceptable hardness and high corrosion resistance performance, which satisfies the demands of the gears working under high speed and corrosive environment. The composite process proposed in this study is considered as a new prospect method due to the multifunction layer design on the gear surface. In summary, a broad range of topics relating to the mechanical properties and nondestructive testing of advanced materials have been collected and presented in this special issue, including both theoretical models and experimental testing methods. These works are expected to be of great interest to scholars in this field.

In the work "The Definition Method and Optimization of Atomic Strain Tensors for Nuclear Power Engineering Materials", a common measure of deformation between atomic scale simulations and the continuum framework is provided and the strain tensors for multiscale simulations are defined. In order to compute the deformation gradient of any atom, the weight function is proposed to eliminate the different contributions within the neighbor atoms which have different distances to atom, and the weighted least squares error optimization model is established to seek the optimal coefficients of the weight function and the optimal local deformation gradient of each atom. The optimization model involves more than 9 parameters. To guarantee the reliability of subsequent parameters identification result and lighten the calculation workload of parameters identification, an overall analysis method of parameter sensitivity and an advanced genetic algorithm are also developed.

"Effect of Chemical Corrosion on the Mechanical Characteristics of Parent Rocks for Nuclear Waste Storage" is a paper with the research interest of nuclear waste disposal repository. Granite was selected as the candidate of parent rocks for nuclear waste storage. The physical and mechanical properties of variation regularity immersed in various chemical solutions were analyzed. Meanwhile, the damage variable based on the variation in porosity was used in the quantitative analysis of chemical damage deterioration degree. Experimental results show that granite has a significant weakening tendency after chemical corrosion. The fracture toughness, splitting tensile strength, and compressive strength all demonstrate the same deteriorating trend with chemical corrosion time. However, a difference exists in the deterioration degree of the mechanical parameters; that is, the deterioration degree of fracture toughness is the greatest followed by those of splitting tensile strength and compressive strength, which are relatively smaller. Strong acid solutions may aggravate chemical damage deterioration in granite. By contrast, strong alkaline solutions have a certain inhibiting effect on chemical damage deterioration. The chemical solutions that feature various compositions may have different effects on chemical damage degree; that is, ions have a greater effect on the chemical damage in granite than ions.

"A Comparative Study for Modeling Displacement Instabilities due to TGO Formation in TBCs of High-Temperature Components in Nuclear Power Plant" reports two numerical simulation methods for modeling displacement instabilities around a surface groove in a metal substrate used in nuclear power plant. The amplitude change in the groove, the downward displacement at the base node, and the groove displacement at the periphery were simulated using ABAQUS to compare the results from two methods, as well as the tangential stress in the elements at the groove base and periphery. The comparison showed that for the tangential stress two methods were in close agreement for all thermal cycles. It is concluded that the present work's numerical simulation scheme worked better with a thinner thermally grown oxide layer than the classic method and could overcome the limitation of thermally grown oxide thickness by simulating any thickness.

In nuclear engineering, nondestructive evaluation and detection of the evaluation of the subsurface damage region are of great importance to ensure the safety of nuclear installations. "Nondestructive Evaluation of Functionally Graded Subsurface Damage on Cylinders in Nuclear Installations Based on Circumferential SH Waves" proposes the use of circumferential horizontal shear waves SH to detect mechanical properties of subsurface regions of damage on cylindrical structures. The regions of surface damage are considered to be functionally graded material and the cylinder is considered to be a layered structure. The Bessel functions and the power series technique are employed to solve the governing equations. By analyzing the $\mathrm{SH}$ waves in the $12 \mathrm{Cr}$-ODS ferritic steel cylinder, which is frequently applied in the nuclear installations, we discuss the relationship between the phase velocities of $\mathrm{SH}$ waves in the cylinder with subsurface layers of damage and the mechanical properties of the subsurface damaged regions. The results show that the subsurface damage could lead to decrease of the $\mathrm{SH}$ waves' phase velocity. The gradient parameters, which represent the degree of subsurface damage, can be evaluated by the variation of the $\mathrm{SH}$ waves' phase velocity. Research results of this study can provide theoretical guidance in nondestructive evaluation for use in the analysis of the reliability and durability of nuclear installations.

In summary, a broad range of topics related to the mechanical properties in nuclear installations and their measurement methods have been collected and presented in this special issue, including both theoretical models and experimental testing methods. These works are expected to be of great interest to scholars in this field.

Yan Yang
Alejandro Clausse
Leon Cizelj
Xing Chen
Parashuram Sahoo



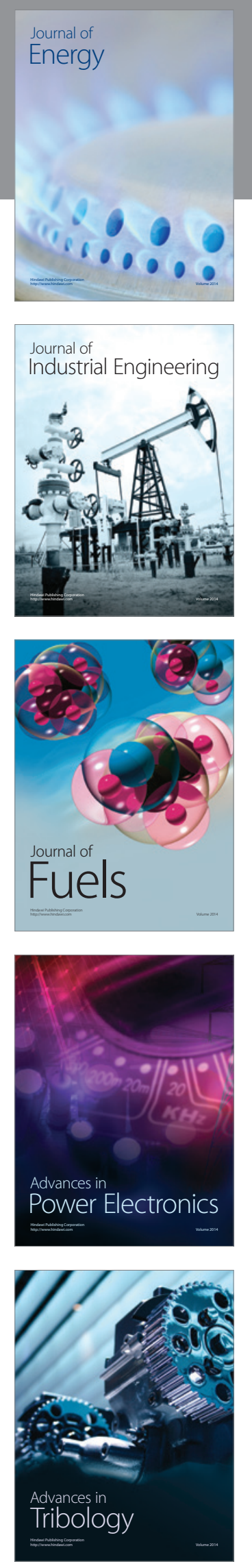
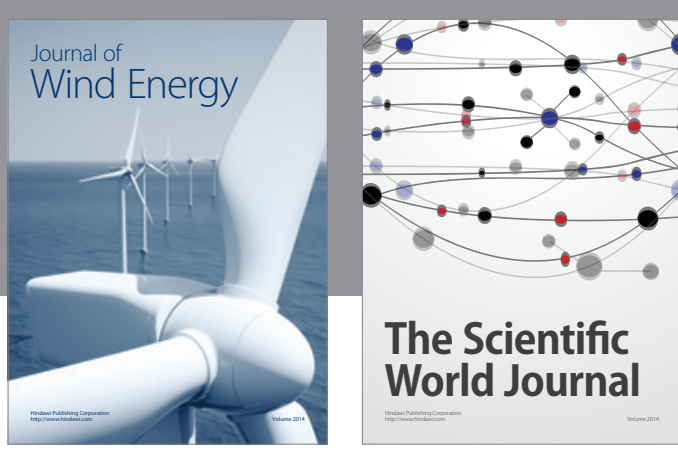

The Scientific World Journal
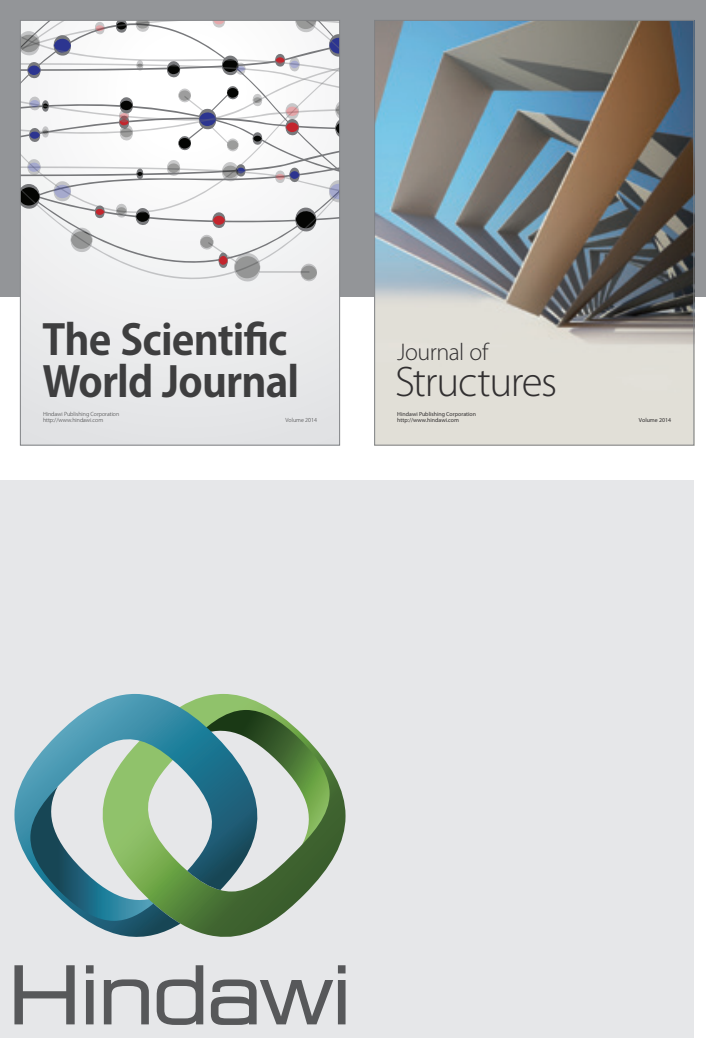

Submit your manuscripts at

http://www.hindawi.com
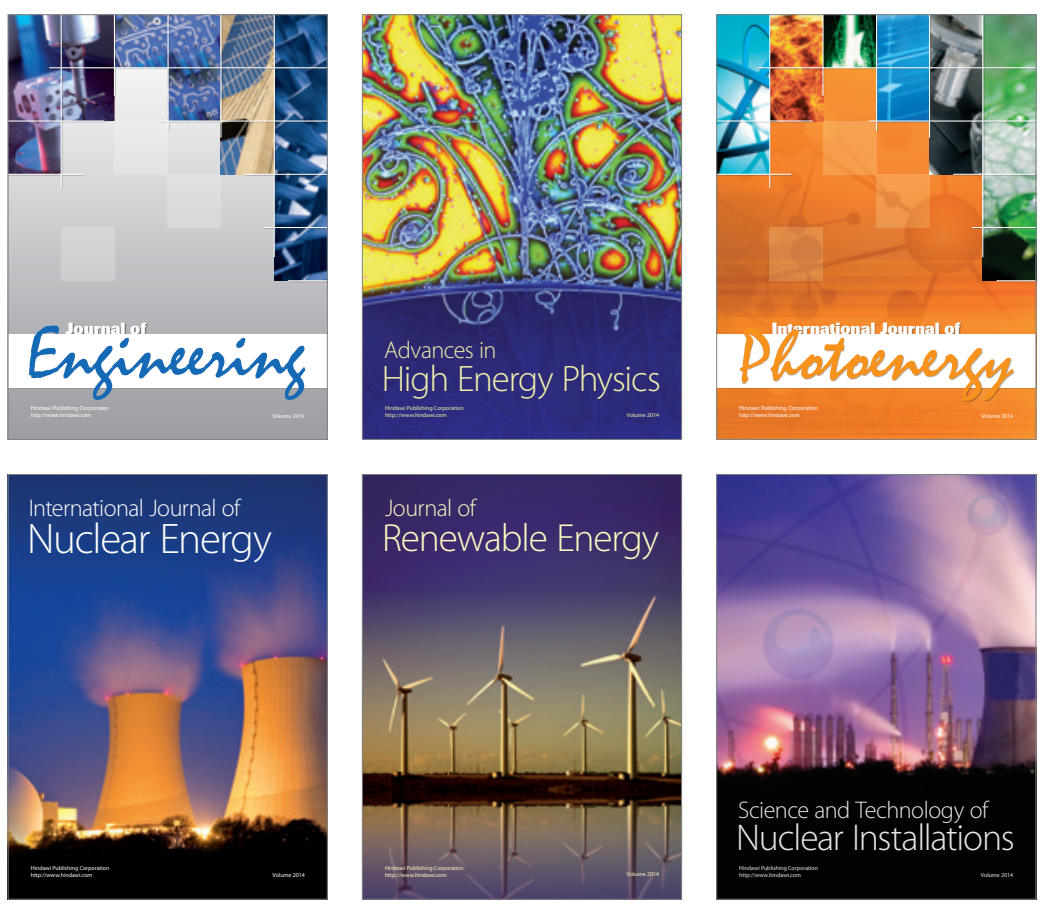
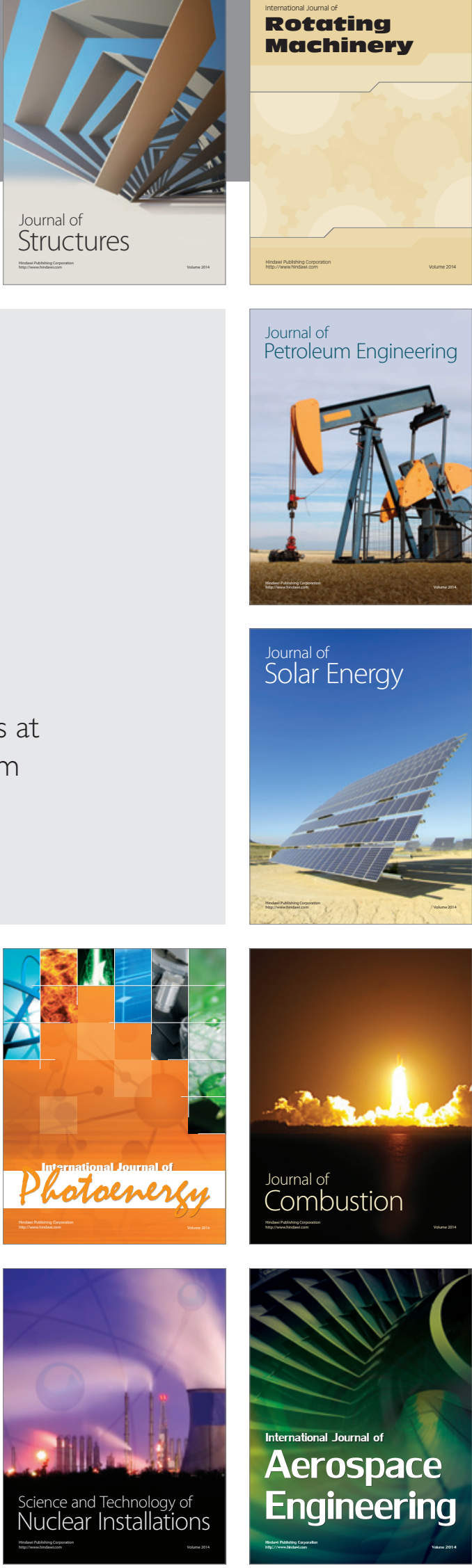